\title{
Künstliches Leben \\ Biologisches Wissen und die Politik der Künste
}

In der griechischen Mythologie war es Aphrodite, die Göttin der Liebe, die aus Mitleid jene elfenbeinerne Statue zum Leben erweckt hat, die den täglichen Liebkosungen ihres Schöpfers Pygmalion nichts als nur die immergleiche kühle Oberfläche entgegenzuhalten hatte. Bei Ovid schließlich wird die Erzählung zur kaiserzeitlichen Parabel der Künstlerschaft: ${ }^{1}$ Das handwerkliche Geschick selbst jenes Bildhauers, der nicht Vorgefundenes nachahmt, ${ }^{2}$ sondern 'Ideen gestaltet, gibt dem Werk zwar seine äußere Form, ${ }^{3}$ doch die Göttin erst füllt diese von innen mit Leben. Diese Vorstellung einer stets unvollkommen bleibenden menschlichen Schöpfung korreliert mit Kernbeständen des bis tief in die Moderne vorherrschenden Wissens von einem Leben, das nur als numinose Gabe begriffen werden kann; eine Gabe, die von innen, und daher unsichtbar, die tote Materie beseelt. ${ }^{4}$ Während die göttliche Schöpfung Lebendiges hervorbringt, ist die Schöpfung des Künstlers eine Totgeburt - sie muss sich stets auf die

1 Vgl. Publius Ovidius Naso: Metamorphosen, übers. und hg. von Erich Rösch, München/ Zürich ${ }^{13} 1992$, Lib. X, V. 243-297, S. 370-372.

2 Denn Pygmalions Werk ist keine mechanisch austrocknende Nachahmung einer natürlich lebendigen Form; er hat vielmehr eine Idealgestalt geschaffen, der keine lebende Frau gleichkommt (»qua femina nasci / nulla potest«, ebd., V. 248f.); er hat damit das Leben noch überboten und in der Überbietung mortifiziert: Pygmalion ist - selbst noch im emphatischen Verständnis des 18. Jahrhunderts - ein Künstler.

3 Das meint hier nicht die imaginative Schau einer platonischen Idee, sondern deren Produktion durch die Einbildungskraft, die Pygmalion als Künstler im emphatischen Sinn erscheinen lässt; dies jedenfalls unter den Vorzeichen der insbesondere seit dem 16. Jahrhundert bis zu Kant gewandelten Idea-Konzeption, die seither weniger den Vorstellungsinhalt als vielmehr das Vorstellungsvermögen selbst bezeichnete. Diesen Wandel macht Erwin Panofsky: IDEA. Ein Beitrag zur Begriffsgeschichte der älteren Kunsttheorie, 2., verb. Aufl., Berlin 1960, S. 33f., plausibel; die nötige Betonung der theoriegeschichtlichen Bedeutung des Konzepts für das 18. Jahrhundert, insbesondere für Goethe, leistet Norbert Christian Wolf: Streitbare Ästhetik. Goethes kunst- und literaturtheoretische Schriften 1771-1789, Tübingen 2001, S. 381-408.

4 Es kann hier nicht näher darauf eingegangen werden, wie sich »die Zeugung - eine jedesmal neue Schöpfung, die stets die Intervention einer externen Kraft erfordert - in die Reproduktion, eine inhärente Eigenschaft eines jeden lebenden Systems, verwandelte.« François Jacob: Die Logik des Lebenden. Von der Urzeugung zum genetischen Code, Frankfurt a.M. 1972, S. 25 und ausführlicher dazu S. 27-36. 
Gestaltung von Oberfläche und Form beschränken. ${ }^{5}$ Trotz aller an der Bildsäule aufgebotenen ästhetischen Finesse bleiben die Küsse trocken, die Umarmungen steif, das Material kalt; denn selbst die kunstfertigste Statue ist am Ende nur Handwerk und bleibt Artefakt. Was die Hände mit größter Geschicklichkeit geschaffen haben, erzeugt Leben nur als Mangel - zumal wenn es emphatisch als Werk zu begreifen ist. ${ }^{6}$ Denn eine Kunst, die ihre eigene Kunstfertigkeit, ihre Künstlichkeit verschleiert, ${ }^{7}$ erzeugt eine Form, die fälschlich Leben verheißt und so beständig enttäuscht.

Da indes auch Lebewesen sich immer nur von außen betrachten lassen, mithin derselben Wahrnehmungsdisposition unterliegen, ${ }^{8}$ können Kunstwerke dennoch zu ihnen (jedenfalls virtuell) in Konkurrenz treten. Die gestaltete Oberfläche ${ }^{9}$ verweist daher nicht nur auf das Defizitäre, sondern erzeugt zugleich einen Anschein, der die größte Stärke des Kunstwerks darstellt: Die Form ist es, die die Vorstellungskraft ihrer Betrachter reizt und lebendige Imaginationen ihrer Innenwelt erzeugt. Die Rede vom slebendigen Kunstwerk v verweist daher nicht einfach auf einen Lesefehler, sondern auf eine kurzschlüssige, wenngleich kulturell gut verankerte Gleichsetzung von zeichenhafter Oberfläche und einem davon eingehüllten inneren Wesen ${ }^{10}$

5 Programmatisch formuliert etwa bei Johann Wolfgang Goethe: Diderots Versuch über die Mahlerey. Uebersetzt und mit Anmerkungen begleitet, in: Propyläen. Eine periodische Schrift, Bd. I.2, Tübingen 1799, S. 1-44, hier S. 10.

6 Nicht erst die Autonomietheorie des 18. Jahrhunderts weist dem Werk eine (zumindest ideale) Stetigkeit zu, die mit der Wandelbarkeit des Lebens unvereinbar scheint. Den meisten Konzeptionen des Werks seither eignet eine solche Tendenz zur Verfestigung; vgl. Carlos Spoerhase: Was ist ein Werk? Über philologische Werkfunktion, in: Scientia Poetica 11 (2007), S. 276-344.

7 "[...] ars adeo latet arte sua«, Ovid: Metamorphosen (wie Anm. 1), Lib. X, V. 252, S. 370.

8 Vgl. Immanuel Kant: Kritik der Urtheilskraft [1790], in: Kant's gesammelte Schriften, hg. von der Deutschen Akademie der Wissenschaften zu Berlin (davor Königlich Preußische Akademie der Wissenschaften, Berlin), Berlin 1900ff., Bd. 5., S. 165-485, hier S. 375.

9 Die geordnete Gestalt wird um 1800 unter geänderten Vorzeichen wieder zum zentralen Merkmal bildender Kunst erhoben. Ein Paralipomenon zu Goethes Propyläen notiert daher unter der Überschrift »Zu bearbeitende Materie“ als Grundproblematik des Künstlers "die Schwierigkeit von dem Formlosen zur Gestalt zu gelangen." (WA I, 47, S. 280; hier und im Folgenden wird mit der Sigle WA, unter Angabe von Abteilungs-, Band- und Seitenzahl, verwiesen auf Goethes Werke, hg. im Auftrage der Großherzogin Sophie von Sachsen, 133 Bde. in 143, Weimar 1887-1919) Zum Gestaltproblem vgl. auch Sabine Schneider: »ein strenger Umriß - Prägnanz als Leitidee von Goethes Formdenken im Kontext der Weimarer Kunsttheorie, in: Goethe-Jahrbuch 128 (2011), S. 98-106.

10 „Gerade das, was ungebildeten Menschen am Kunstwerk als Natur auffällt, das ist nicht Natur (von außen), sondern der Mensch (Natur von innen)." (Johann Wolfgang Goethe: Maximen und Reflexionen, Nr. 1076, in: Ders.: Sämtliche Werke nach Epochen seines Schaffens. Münchner Ausgabe, hg. von Karl Richter in Zusammenarbeit mit Herbert G. Göpfert u.a., München 1985-1998, im Folgenden zitiert mit der Sigle MA, Bd. 17, S. 899). 
Lebendigkeit meint daher in Bezug auf einzelne Werke den habitualisierten Umgang mit einer optischen Täuschung, sie ist das jahrhundertelang gut gepflegte metaphorische Qualitätsprädikat der Künste. ${ }^{11}$

Es liefert zwar bereits Ovids Verwandlungsszene das grundlegende Modell für das (stets prekäre) Verhältnis von Kunst und Leben, auf das die ästhetischen Debatten und künstlerischen Praktiken seither sich vorzugsweise stützen; doch die moderne Biologie, die sich (rückblickend) seit dem 18. Jahrhundert als Lebenswissenschaft zu etablieren begonnen haben wird, ${ }^{12}$ scheint dieses Verhältnis neu akzentuiert zu haben. ${ }^{13}$ Wenn im Folgenden den Figurationen von künstlichem Leben, einer "vita aesthetica kunstvoller Zeichenpraktiken ${ }^{14}{ }^{14}$ in gerade jenem Zeitraum nachgegangen werden soll, in dem sich das Wissen vom Leben durch die Verbreitung und den Einfluss von organologischen Formations- und epigenetischen Generationsmodellen zu wandeln begann, ${ }^{15}$ dann soll dies mit grundsätzlicher Skepsis gegenüber einfachen, auf simplen Koinzidenzen gründenden Erklärungen geschehen. $\mathrm{Zu}$ fragen sein wird vielmehr nach den Akteuren und Medien, über die Verbindungen zwischen den beiden Diskursbereichen hergestellt und unter-

11 Vgl. Peter Brandes: Leben die Bilder bald? Ästhetische Konzepte bildlicher Lebendigkeit in der Literatur des 18. und 19. Jahrhunderts, Würzburg 2013, S. 9-26.

12 Eine solche genealogische Sichtweise auf die Geschichte der vorwiegend im Singular auftretenden 'Lebenswissenschaft wird auch von aktuellen Einführungen formuliert; pointiert etwa von Thomas Junker: Geschichte der Biologie. Die Wissenschaft vom Leben, München 2004, S. 7: "Zur Entstehung der Biologie als übergreifende Wissenschaft von den Lebewesen kam es erst im 19. Jahrhundert." Gegen solche Narrative des Werdens betont Foucault die Brüche, die erst die Opposition zwischen Lebendigem und NichtLebendigem ermöglicht haben; vgl. Michel Foucault: Die Ordnung der Dinge. Eine Archäologie der Humanwissenschaften, Frankfurt a.M. 1974, S. 279-287.

13 Daher erfordert der moderne Roman eine "Theorie des Lebens", mit der eine "Wende im Wissen der Literatur" verbunden ist, während ästhetische Formtheorie "vom Leben her gedacht Bedürfnis nach Form und von der Ästhetik aus gesehen eines nach Biologie ist." Rüdiger Campe: Form und Leben in der Theorie des Romans, in: Vita aesthetica. Szenarien ästhetischer Lebendigkeit, hg. von Armen Avanessian, Winfried Menninghaus und Jan Völker, Zürich/Berlin 2009, S. 193-211, hier S. 195.

14 Armen Avanessian/Winfried Menninghaus/Jan Völker: Einführung, in: Vita aesthetica (wie Anm. 13), S. 7-11, hier S. 11. Es wird damit der terminologische Übergang von der "Lebendigkeit« zum "Leben« im 18. Jahrhundert bezeichnet, der - etwas hochgegriffen vielleicht - zugleich einen »Paradigmenwechsel der Ästhetik selbst« markiere (ebd., S. 10).

15 Dies geschieht im Rahmen des Übergangs "von der Naturgeschichte zu einer Geschichte der Natur", von dem Wolf Lepenies: Das Ende der Naturgeschichte. Wandel kultureller Selbstverständlichkeiten in den Wissenschaften des 18. und 19. Jahrhunderts, Frankfurt a.M. 1978, S. 50, spricht. 
halten werden konnten, sowie nach den theoretischen Verlusten und Überschüssen, die von den Transferhandlungen verursacht wurden. ${ }^{16}$

I.

Das Erstaunliche am Pygmalion-Mythos ist, dass er den Normalfall der Täuschung des Betrachters durch eine Überwältigung des Künstlers übersteigt, indem das Werk sogar seinen eigenen Schöpfer zu betrügen vermag. Seit der frühen Aufklärung findet man vermehrt Zeichen jener Verwunderung, durch die Pygmalion in die ästhetischen Debatten zurückkehren und zum Sinnbild einer »neue[n] Auffassung vom Künstler«, zum »Exempel für die belebende Kraft ästhetischer Imagination « werden konnte. ${ }^{17}$ Im Vorübergehen zwar, doch nicht ohne ebenjene Überraschung bemerkt dann auch Johann Jacob Bodmer, dass die von gelungenen Werken gereizte Einbildungskraft offenbar selbst die Erinnerung an die eigene Tätigkeit tilgen und noch dem Schöpfer des Toten Lebendigkeit vorgaukeln kann:

Wer sollte vor dergleichen Entzückung und Betrug besser verwahret gewesen seyn als der Meister und Urheber dieses Bildes, der den rohen und leblosen Marmor in seiner ungestalteten Figur gesehen, mit seiner Hand ihm eine bessere Form und Bildung, ein Gliedmaß, ein Lineament nach dem andern gegeben, und noch jetzo lauter Kälte und eine leblose Stille an dieser menschlichen Gestalt fühlete? Alle diese Betrachtungen, welche den Betrug so schwer macheten, zernichtete die Kunst durch den vollkommenen Ausdruck des Lebens, welches sie so geschickt nachzuahmen wußte. ${ }^{18}$

Der in der Form codierte 'Ausdruck des Lebens löscht die Erinnerung an die Abstammung vom »leblosen Marmor . Pygmalion jedenfalls kann sich nur mit Mühe und nur durch wiederholte prüfende Berührungen versi-

16 Mögliche Verbindungen von ästhetischer Theorie und biologischer Vererbungslehre im Konzept der Generativität zeigen Ohad Parnes/Ulrike Vedder/Stefan Willer: Das Konzept der Generation. Eine Wissenschafts- und Kulturgeschichte, Frankfurt a.M. 2008, S. $120-149$, auf.

17 Helmut Pfotenhauer: Pygmalion. Heinses Hermeneutik lebendiger Bilder, in: Ders.: Um 1800. Konfigurationen der Literatur, Kunstliteratur und Ästhetik, Tübingen 1991, S. 27 56, hier S. 27.

18 Johann Jacob Bodmer: Critische Betrachtungen über die Poetischen Gemählde der Dichter. Mit einer Vorrede von Johann Jacob Breitinger, Zürich 1741, S. $42 \mathrm{f}$. 
chern, ${ }^{19}$ dass das von ihm aus roher Materie Geformte nichts weiter ist als ein Kunstwerk. Es ist genau dieser prekäre Moment der Selbstversicherung, der zum Streitpunkt werden und mit der durch ihn problematisierten Rede vom lebendigen Kunstwerk zugleich eine Krise des rhetorischen (Regel-) Systems der Künste anzeigen wird. ${ }^{20}$ Obwohl man nicht umhin kommt, für das Ende des 18. Jahrhunderts eine Affinität der Künste zur Biologie zu konstatieren, wird man nicht von einer einfachen Übergängigkeit der Künste in das seit Buffon ethologisch reformulierte Regelsystem des Lebens und der Zeugung sprechen können. ${ }^{21}$ Denn die als Befreiung gefeierte Lossprechung der Kunst aus Wirkungs- und Repräsentationszusammenhängen erzeugt einen folgenreichen double bind: Um das Kunstwerk freizuspielen, wird es als >organisch strukturiertes Analogon der Natur und zugleich emphatisch als Erzeugnis eines Künstlerindividuums definiert. ${ }^{22}$ Entrüstet über die beide Positionen unterlaufende Tendenz von Denis Diderots Essais sur la peinture, das Kunstwerk der einfachen Natur anzunähern, lässt Goethe in seiner kommentierten Übersetzung die Natur selbst als "personificirte, göttliche Gestalt« auftreten und das Wort ergreifen:

Die Tradition sagt: daß brutale Menschen gegen plastische Meisterwerke von sinnlichen Begierden entzündet wurden; die Liebe eines hohen Künstlers aber zu seinem trefflichen Werk ist ganz anderer Art; sie gleicht der frommen, heiligen Liebe unter

19 Johann Gottfried Herder wird diese Geste der Versicherung in ihr Gegenteil umwenden, indem er die visuelle Körperimagination durch das Betasten um eine Dimension erweitert und damit die Vorstellung der Wirklichkeit noch annähert. Er betont, »daß das Gesicht uns nur Gestalten, das Gefuihl allein, Körper zeige: da/s Alles, was Form ift, nur durchs tastende Gefühl, durchs Gesicht nur Fläche, und zwar nicht körperliche, sondern nur sichtliche Lichtfläche erkannt werde." (Hervorhebung im Original) Es kommt dabei auf die theoriegeschichtlich neue Annahme an, dass "die fühlende Hand in besonderer Weise geeignet sei, Leben nicht nur ’herzustellen`, sondern am Kunstwerk zu erfahren«. Inka Mülder-Bach: Im Zeichen Pygmalions. Das Modell der Statue und die Entdeckung der »Darstellung« im 18. Jahrhundert, München 1998, S. 69.

20 Damit wird die Vorstellung des lebendigen Kunstwerks indes keineswegs abgeschafft. Noch zu Beginn des 20. Jahrhunderts kann das Kunstwerk ganz unproblematisch als ein »durch und durch lebendiges Gebilde« begriffen werden; vgl. Wilhelm Waetzoldt: Das Kunstwerk als Organismus. Ein ästhetisch-biologischer Versuch, Leipzig 1905, S. 18.

21 Vgl. die Pointierung des Unterschieds in den Systematiken von Linné und Buffon bei Werner Michler: Klassifikation und Naturform. Zur Konstitution einer Biopoetik der Gattungen im 18. Jahrhundert, in: Gattungs-Wissen. Wissenspoetologie und literarische Form, hg. von Michael Bies, Michael Gamper und Ingrid Kleeberg, Göttingen 2013, S. 35-50, hier S. 40f.

22 Siehe auch Daniel Ehrmann: Bildverlust oder Die Fallstricke der Operativität. Autonomie und Kulturalität der Kunst in den Propyläen, in: Klassizismus in Aktion. Goethes Propyläen und das Weimarer Kunstprogramm, hg. von dems. und Norbert Christian Wolf, Wien/ Köln/Weimar 2016, S. 123-173. 
Blutsverwandten und Freunden. Hätte Pygmalion seiner Statue begehren können, so wäre er ein Pfuscher gewesen, unfähig eine Gestalt hervorzubringen, die verdient hätte, als Kunstwerk oder als Naturwerk geschätzt zu werden. ${ }^{23}$

Zwar begibt sich hier die Personifikation der Natur auf metaphorisch schwieriges Terrain, wo nicht zuletzt Zeugung und Verwandtschaft in gefährliche Nähe geraten, ${ }^{24}$ und sie scheint sich ebenfalls in dem problematischen Verhältnis von Natur und Kunst zu verstricken. Jedoch macht sie eines dabei deutlich: Statuenliebe ist eine Brutalität, die keinem swahren Künstler unterlaufen kann; betroffen sind von einer solchen Verwechslung von Sinneswahrnehmung und Affektwirkung höchstens »die Jugend, das Frauenzimmer, ein großer Teil der nordischen KunstLiebhaber « ${ }^{25}$ Lebendig kann Kunst nur scheinen, nie eigentlich sein, ${ }^{26}$ und sie läuft Gefahr, erneut und endgültig einer Natur zu unterliegen, die durch das Konzept selbstreproduzierender Organismen vom Numinosen freigespielt wird. Denn wie können Abgeschlossenheit implizierende Werke mit solchen Diskursen des Lebendigen verschnitten werden? Insbesondere wenn es sich dabei um Kunstwerke handelt, die immer auch Formen des Handwerks sind? Können die sinneren Gesetze des Lebens، eine Entsprechung im Bereich der Kunst finden? Solche Fragen scheinen viele der gattungspoetologischen Debatten um 1800 zu befeuern und die Suche nach tauglichen Konzepten einer 'Naturpoesie zu motivieren. Dabei erscheint der Glaube an die Lebendigkeit des Kunstwerks nunmehr als Exklusionskriterium, über das die Vertreter einer veränderten Auffassung vom Leben in der Kunst sichtbar werden, die weniger an der Frage nach der Lebendigkeit einzelner Werke interessiert sind, als an dem, was der Blumenbach-Schüler Carl Friedrich Kielmeyer 1793 »das Leben der Gattung « genannt hat. ${ }^{27}$

23 Goethe: Diderots Versuch über die Mahlerey (wie Anm. 5), S. 20.

24 Zur daran geknüpften Angst der Hygieniker vor Degeneration vgl. Philipp Sarasin: Reizbare Maschinen. Eine Geschichte des Körpers 1765-1914, Frankfurt a.M. 2001, S. 433441.

25 Johann Wolfgang Goethe: [Anzeige] Propyläen. Eine periodische Schrift, herausgegeben von Goethe. Ersten Bandes Erstes und Zweites Stück, Zweiten Bandes Erstes Stück, Tübingen 1799 in der Cottaschen Buchhandlung, in: MA 6.2, S. 131-139, hier S. 132. Vgl. auch Ders.: Der Versuch als Vermittler von Objekt und Subjekt, in: MA 4.2, S. 321-332, hier S. 321, und Ders.: Ueber Wahrheit und Wahrscheinlichkeit der Kunstwerke, in: Propyläen. Eine periodische Schrift, Bd. I.1, Tübingen 1798, S. 55-65, bes. S. 60-62.

26 Vgl. die frühe Polemik in Goethes Brief an F. H. Jacobi, 21.8.1774, WA IV, 2, S. 186f. Als Ergebnis einer »Vereinigung von Object und Subject« wird das slebendige Werk hingegen symbolische Form (vgl. Goethe an C. L. F. Schultz, 18.9.1831, WA IV, 49, S. 82).

27 Carl Friedrich Kielmeyer: Ueber die Verhältniße der organischen Kräfte unter einander in der Reihe der verschiedenen Organisationen, die Geseze und Folgen dieser Verhält- 
II.

Die Vermutung liegt nahe, dass die sowohl in den ästhetischen wie (im weiteren Sinn) naturkundlichen Debatten des ausgehenden 18. und beginnenden 19. Jahrhunderts zur Zentralkategorie avancierte Form interdiskursive Funktion ausübt. ${ }^{28}$ Jedenfalls aber scheinen die Form und die Frage nach formativen Prozessen zentrale Semantiken beider Diskurse in ähnlicher Weise zu strukturieren: Organismen sind ebenso wenig ohne spezifische Lebensform ${ }^{29} \mathrm{zu}$ denken wie Kunst ohne $>$ Werkform ${ }^{30}{ }^{30}$ Es scheint also nur der Kategorie der Form zu gelingen, ihre Beschreibungsobjekte der Ästhetik wie der Biologie - das sind nicht nur Einzelwesen und Werke, sondern künstlerische wie biologische Gattungen - voneinander zu trennen und in hierarchische, später genealogische Ordnungen zu versetzen. In diesem Sinn sind bereits die Tableaus der taxonomischen Naturgeschichte und die klassizistische Hierarchie der Kunstgattungen ${ }^{31}$ ohne differierende Formen undenkbar; diese gewinnen aber neue Bedeutung für die vergleichende

niße. Eine Rede den 11ten Februar 1793 am Geburtstage des regierenden Herzogs Carl von Wirtemberg im großen akademischen Hörsale gehalten, neuer unv. Abdr., Tübingen 1814, S. 7.

28 Vgl. grundlegend zu Begriff und Konzept Jürgen Link: Elementare Literatur und generative Diskursanalyse (mit einem Beitrag von Jochen Hörisch und Hans-Georg Pott), München 1983, bes. S. 14f.

29 Der Begriff der Lebensform soll hier darauf hinweisen, dass die tatsächliche Bewegtheit und Varianz lebendiger Gestalten im Zuge ihrer Diskursivierung in Normalformen des Lebens stillgestellt werden. Bereits in den Debatten um 1800 erscheint Leben stets als geformtes Leben. Diese Differenz von zoé und bios wird von Giorgio Agamben: LebensForm, in: Gemeinschaften. Positionen zu einer Philosophie des Politischen, hg. von Joseph Vogl, Frankfurt a.M. 1994, S. 251-257, aufgegriffen und politisch radikalisiert.

${ }^{30} \mathrm{Zu}$ dieser Zentralkategorie der spätaufklärerischen Populärphilosophie vgl. Salomon Maimon: Ueber die Ästhetik, in: Ders.: Streifereien im Gebiete der Philosophie. Erster Theil, Berlin 1793, S. 131f. Zur Bedeutung der Form in keineswegs nur klassizistischen Poetiken insbesondere des llangen 19. Jahrhunderts vgl. Dieter Burdorf: Poetik der Form. Eine Begriffs- und Problemgeschichte, Stuttgart/Weimar 2001.

31 Die an der Pariser Académie royale mit einiger Verbindlichkeit formulierte bildkünstlerische Gattungshierarchie folgt wie die biologische dem Prinzip der Komplexitätssteigerung; vgl. Werner Busch: Das sentimentalische Bild. Die Krise der Kunst im 18. Jahrhundert und die Geburt der Moderne, München 1993, S. 22-24. Bei Anton Raphael Mengs: Gedanken über die Schönheit und über den Geschmack in der Malerey, hg. von J. Caspar Füeßlin, 3. Aufl., Zürich 1771, wird die Hierarchie der Gattungen zu einer Hierarchie des Geschmacks modifiziert, die bereits einen zeitlichen Index aufweist, eine Geschichte hat. Daher »ist die Kunst und ihr Geschmak gestiegen und gefallen. Endlich ist sie gar zu grunde gegangen da die Künstler aus Unwissenheit anfingen nur nach der Gewohnheit der Handwerker zu arbeiten« (ebd., S. 47). 
Anatomi $^{32}$ und die bald akademisch werdenden kunst- und literaturhistorischen Unternehmungen am Beginn des 19. Jahrhunderts.

Wenn Form eine solche zentrale Funktion ausübt, dann muss die von der Verzeitlichung ausgehende Bedrängnis in Vorstellungen der Weitergabe und Tradition aufgehoben werden; es kommt daher bereits vor der Etablierung eines gesicherten Wissens von der konkreten >Mechanik der Reproduktion zu Imaginationen und Spekulationen über die Rolle der Vererbung. ${ }^{33}$ Im Zuge dieser Spekulationen erhält der Organismus eine Tiefendimension, ${ }^{34}$ die nicht mehr nur seine oberflächliche Erscheinung funktional erklärt, sondern zugleich deren autonome Fortzeugung verständlich machen soll. Dieses spannungsvolle Verhältnis von Oberfläche und Tiefenschicht wird dann bei Kant in ein auch für die Kunst folgenreiches Konzept gegossen werden: "Ein organisirtes Wesen [...] besitzt in sich bildende Kraft und zwar eine solche, die es den Materien mittheilt, welche sie nicht haben (sie organisirt): also eine sich fortpflanzende bildende Kraft, welche durch das Bewegungsvermögen allein (den Mechanism) nicht erklärt werden kann. ${ }^{35}$ Die Schachteln und Keime der Präformationisten sind längst verschwunden, stattdessen vermittelt Kant Buffons 'moule intérieur mit Blumenbachs 'nisus formativus` und legt Nachdruck auf die reflexiv und transitiv organisierende Kraft der Wesen, die auch ein unberechenbar bleibendes Moment der Dynamik in die Gestaltwerdung einbringt. ${ }^{36}$ Deshalb geht die Reproduktion einher "mit schicklichen Abweichungen, die

32 Die an einer räumlichen Ordnung der Formen orientierte Naturgeschichte gerät im Laufe des 19. Jahrhunderts vonseiten einer die formalen Trennungen subvertierenden vergleichenden Anatomie oder einer an der ’Oeconomier des Verhaltens interessierten vergleichenden Naturgeschichte unter Druck. Vgl. dazu exemplarisch Johann Friedrich Blumenbach: Versuch einer vergleichenden Physiologie der warmblütigen, lebendig gebährenden und Eierlegenden, Thiere, in: Ders.: Kleine Schriften zur vergleichenden Physiologie und Anatomie und Naturgeschichte gehörig. Übers. und hg. von Johann Gottfried Gruber, Leipzig 1800, S. 1-60; eine Verbindung zwischen Verhalten bzw. Psychologie und Entwicklungsstufe schlägt Georg Friedrich von Jäger: Beiträge zur vergleichenden Naturgeschichte der Thiere und des Menschen. In zwey Reden, Stuttgart 1830, vor.

33 Es sucht auch Buffon, »die Mechanik zu erklären, deren sich die Natur zu Hervorbringung seines gleichen bedient«. Vgl. [George-Louis Leclerc de Buffon:] Allgemeine Historie der Natur nach allen ihren besondern Theilen abgehandelt [...], übers. von Bartholomäus Joachim Zink, Bd. 1.2, Hamburg/Leipzig 1750, S. 22.

34 Vgl. Jacob: Die Logik des Lebenden (wie Anm. 4), S. $79 f$.

35 Kant: Kritik der Urtheilskraft (wie Anm. 8), S. 374.

36 In dieser Bedeutung erscheint der Begriff der Kraft auch in den ästhetischen Debatten seit Baumgarten und Herder; vgl. Christoph Menke: Kraft. Ein Grundbegriff ästhetischer Anthropologie, Frankfurt a.M. 2008, S. 46-66. 
die Selbsterhaltung nach den Umständen erfordert. ${ }^{37}$ Diese Dynamik der Reproduktionskraft überträgt Carl Friedrich Kielmeyer auf die Entwicklung der Gattungen; ${ }^{38}$ er weitet die an den Individuen beobachtbaren formbildenden Prozesse auch auf nur vorgestellte Gattungswesen aus und schlägt damit unwillkürlich den Bogen ins Feld der Kunst: Wenngleich das biologische Wissen den einzelnen Werken ihr Leben entzog, so eröffnet es dadurch nur die Perspektive auf die Lebendigkeit einer weitaus größeren Einheit - der Gattung.

Leben erscheint - in der Kunst wie in der Natur - nie selbst, sondern immer nur in Vermittlung durch Zeichen, und diese sind, nach der Terminologie von Charles Sanders Peirce, indexikalischer Natur. ${ }^{39}$ Leben tritt daher immer nur im Nachvollzug einer Spur von Bewegung, von Formveränderung in Erscheinung. ${ }^{40}$ Erst wenn nicht einzelne Kunst- oder 'Naturwerker isoliert und momenthaft betrachtet werden; ${ }^{41}$ wenn nicht Zustände, sondern das Werden von Formen, deren ontogenetische, später auch phylogenetische Entwicklung in den Blick geraten; wenn Formen einer spezifischen Verzeitlichung unterworfen werden, wird aus der Lebendigkeit des Kunstwerks mehr als eine Metapher im rhetorischen System der Künste. Die Formen erscheinen dann als Emanationen des Lebens einer Gattung. Die Konzepte von Kunst und Natur überschneiden sich damit in einem Lebenswissen, ${ }^{42}$ das beide Kollektivsingulare als Diskursobjekte verän-

37 Kant: Kritik der Urtheilskraft (wie Anm. 8), S. 374.

38 Vgl. Kielmeyer: Ueber die Verhältniße der organischen Kräfte (wie Anm. 27), S. $40 f$.

39 Vgl. Charles Sanders Peirce: Phänomen und Logik der Zeichen, hg. und übers. von Helmut Pape, Frankfurt a.M. 1983, S. 65.

40 Der Diskurs um 1800 kann sich einer bestimmten Zielgerichtetheit nicht ganz entschlagen. Jedenfalls werden Leben und Entwicklung in annähernd gleicher Bedeutung verwendet; vgl. exemplarisch Kielmeyer: Ueber die Verhältniße der organischen Kräfte (wie Anm. 27).

41 Es ist bezeichnend, dass um 1800 Natur und Kunst streng und grundsätzlich geschieden werden sollen, die einzelnen Phänomene beider Bereiche aber in einer signifikanten Übertragung des Werkbegriffs einander auf zweiter Ebene angenähert werden. Goethe spricht einige Jahre lang gehäuft von 'Naturwerken<, die den Kunstwerken analog beschrieben werden können. Vgl. etwa: „Ein ächtes Kunstwerk bleibt, wie ein Naturwerk, für unsern Verstand immer unendlich; es wird angeschaut, empfunden; es wirkt, es kann aber nicht eigentlich erkannt, vielweniger sein Wesen, sein Verdienst mit Worten ausgesprochen werden." (Johann Wolfgang Goethe: Ueber Laokoon, in: Propyläen. Eine periodische Schrift, Bd. I.1, Tübingen 1798, S. 1-19, hier S. 1)

42 Kurz gefasst: "Die Kenntnis von den Lebewesen bezieht die Vorstellungen vom Leben und die einer geschichtlichen Entwicklung mit ein."Jacob: Die Logik des Lebenden (wie Anm. 4), S. 21. 
dert. ${ }^{43}$ Denn in der Verzeitlichung der Ordnungen drängt sich die Frage nach Entwicklung, Veränderung und Weitergabe auf. Das Nichtwissen, in das die Mechanismen und die Regeln der Vererbung eingehüllt waren, ${ }^{44}$ stellt nicht zuletzt deshalb ein Moment größter Virulenz dar, weil es die Frage nach der Herkunft zeitgenössischer Formen impliziert und damit die Gattungen nicht nur der Lebewesen, sondern auch der Künste mit einem zeitlichen Index versieht. Es ist auffällig und kann vielleicht als Reaktion auf eine Zwangslage verstanden werden, dass die folgenreichsten Versuche, die poetischen Gattungen durch eine ontologische Begründung transhistorisch zu stabilisieren und sie aus dem ephemeren Zustand der Zeitgenossenschaft zu befreien, ${ }^{45}$ gerade zu jener Zeit unternommen wurden, als sie nach dem Vorbild der biologischen Gattungen eine Geschichte erhielten, oder sich zumindest in eine (selektive) Traditionslinie einzufügen begannen, und so die Trajektorien bestimmter Merkmale sichtbar wurden. ${ }^{46}$

III.

An der Wende zum 19. Jahrhundert sind es daher längst nicht mehr die Götter, es sind die Musen und unter diesen vor allem Klio, die den Kunstwerken Leben einhauchen. Denn durch die Historisierung der Künste,

${ }^{43}$ Diese Objekte müssen sich mit den sie konstituierenden Diskursen sowie mit deren Beschreibung verändern; vgl. Michel Foucault: Archäologie des Wissens, übers. von Ulrich Köppen, Frankfurt a.M. 1973, S. 199f. Es wird eine neue Kunst entstanden sein, wenn Werke als Vertreter biologieanaloger Gattungen verstanden werden. Indem sie Objekt von Zurichtung und Beobachtung werden, erscheinen biologische wie künstlerische Gattungen um 1800 daher als ähnliche Vertreter von spezifischem Nichtwissen wie die von Hans-Jörg Rheinberger: Experimentalsysteme und epistemische Dinge. Eine Geschichte der Proteinsynthese im Reagenzglas, Göttingen 2001, S. 24-26, beschriebenen repistemischen Dinger.

44 Einen historischen Überblick geben Hans-Jörg Rheinberger/Staffan Müller-Wille: Vererbung. Geschichte und Kultur eines biologischen Konzepts, Frankfurt a.M. 2009.

$45 \mathrm{Zu}$ der (jedenfalls scheinbaren) Frontstellung von ’klassizistischer Gattungspoetik und ihrer ,Überwindung insbesondere durch Friedrich Schlegel vgl. Peter Szondi: Von der normativen zur spekulativen Gattungspoetik, in: Ders.: Poetik und Geschichtsphilosophie II, hg. von Wolfgang Fietkau, Frankfurt a.M. 1974, S. 7-183, bes. S. 131-151.

$46 \mathrm{Zu}$ den zwischen Überzeitlichkeit und Temporalisierung im Unentschieden bleibenden Ordnungsversuchen der frühen Kunstgeschichte vgl. auch Daniel Ehrmann: Ordnen und lenken. Kunstgeschichte und Kunsttheorie in Meyers Beiträgen zu den Propyläen, in: Johann Heinrich Meyer - Kunst und Wissen im klassischen Weimar, hg. von Alexander Rosenbaum, Johannes Rößler und Harald Tausch, Göttingen 2013, S. 255-273, bes. S. 266-273. 
wie sie spätestens von der französischen Querelle des Anciens et des Modernes angestoßen und dann vor allem von Johann Joachim Winckelmann auch systematisch betrieben wurde, erhielten Kunstwerke als generische Erscheinungsformen einen zeitlichen Index. Werke und Gattungen wurden so ihrer diskreten Individualität enthoben und in die Erzählung einer Entwicklungsgeschichte integriert. Insbesondere in seiner 1764 erschienenen Geschichte der Kunst des Alterthums hat sich Winckelmann an einer stiltypologischen Ordnung versucht, durch die (antike) Kunst zugleich systematisiert und historisiert wurde. In seiner zugleich chrono- und genealogischen Betrachtung der Werke bemerkt er 'Wachsthum<, 'Blüthe und >Verfall (vgl. GKA, 213-248) ${ }^{47}$ und leitet daraus die typische Verlaufsform der sich wiederholenden "Stuffen des Wachsthums" (GKA, 223) in der Kunstentwicklung ab, die "auf das Wesen derselben" (GKA, 213) abzielt und sich einem metaphorischen Gerüst verdankt, das sich nicht zuletzt aus dem Bildbereich biologischer Prozesse speist. ${ }^{48}$ Bereits früh formuliert der Kunsthistoriker die Idee einer organischen Verfasstheit des Werks: "Je mehr Einheit aber in der Verbindung der Formen, und in der Ausfließung einer aus der andern ist, desto größer ist das Schöne des Ganzen« (GKA, 152). Schönheit, als Analogon der Funktionalität des biologischen Organismus, ist ein Resultat interdependenter Formen, die sich gegenseitig bedingen und sich sogar wechselweise zu erzeugen scheinen, jedenfalls aus einander rausfließen Dass es sich hierbei um mehr als eine taugliche Metaphorik handelt, um die spröde Systematik seiner Untersuchung stilistisch annehmlicher werden zu lassen, macht Winckelmann in wiederholt eingeschalteten theoretischen Reflexionen deutlich. Dreißig Jahre vor Kielmeyer wagt er den Schritt vom lebendigen Kunstwerk zum `Leben der Gattung«, indem er den biologischen Entwicklungsprozess auf stilistische Veränderungen einzelner Genres überträgt. Die Kunst scheint dabei ein von den Künstlern seltsam getrenntes Eigenleben zu entwickeln: Denn es musste auch »die Kunst, in welcher, wie in allen Wirkungen der Natur, kein fester Punct zu denken ist, da sie nicht

47 Hier und im Folgenden wird mit der Sigle GKA und Seitenzahl verwiesen auf Johann [Joachim] Winckelmann: Geschichte der Kunst des Alterthums, Dresden 1764.

48 Zugleich wird diese biologische Wachstumsanalogie mit der Organisation der dramatischen Handlung in fünf Akten verglichen. "Denn so wie eine jede Handlung und Begebenheit fünf Theile, und gleichsam Stufen hat, den Anfang, den Fortgang, den Stand, die Abnahme, und das Ende, worinn der Grund lieget von den fünf Auftritten oder Handlungen in Theatralischen Stücken, eben so verhält es sich mit der Zeitfolge derselben [gemeint ist die Kunst der Griechen]: da aber das Ende derselben außer die Gränzen der Kunst gehet, so sind hier eigentlich nur vier Zeiten derselben zu betrachten« (GKA, 213f.). 
weiter hinausgieng, zurück gehen« (GKA, 235). Die klassischen Werke der Kunst werden damit aus ihrer ästhetischen Eigenzeit in die prozessuale Zeitlichkeit des Lebens überführt.

Winkelmanns Geschichte der Kunst des Alterthums war ein Diskursereignis, das größte Auswirkungen nicht nur auf wesentliche ästhetische und kunsttheoretische Debatten der folgenden Jahrzehnte hatte, ${ }^{49}$ sondern offenkundig auch das Modell für die Übertragung genealogischer Entwicklungsmodelle auf die Kunst darstellte. Es ist für die Bestimmung des Charakters der hier aufgerufenen Wissensbestände aufschlussreich, dass es bei Winckelmann letztlich keine Künstler sind, die den stilistischen Wandel bewirken. ${ }^{50}$ Er ist nicht an den Zäsuren, an herausragenden Individuen oder singulären Ereignissen interessiert, sondern an anhaltenden formativen Prozessen. Daher sind es in der Geschichte der Kunst des Alterthums nicht die Künstler, die Formen hervorbringen und Gattungen evolvieren lassen, sondern Kulturen. Die Historisierung der Werke wird so gepaart mit einer Naturalisierung der Gattungen, auf die sie bezogen sind. Damit ist bereits das Dilemma bezeichnet, dem sich auch die Dichtungstheorie am Übergang zum 19. Jahrhundert zu stellen haben wird: Zwar wird Werken und Gattungen eine inhärente, gestaltwandelnde Kraft und ein der organischen Natur analoges Eigenleben zugestanden, wodurch sie jene diskursiven Festschreibungen der auf sowohl deskriptiver wie präskriptiver Konvention beruhenden rhetorischen Gattungssystematik seit der Renaissance aufzubrechen geeignet scheinen; doch bleiben zugleich die sichtbaren Zeichen dieser Kraft auf das Austreiben der oberflächlichen Materie gemäß einer inneren Form beschränkt. ${ }^{51}$ Wie die Bildungsgeschichte tierischer Körper ist die Entwicklungsgeschichte der Kunstformen somit von einem neuplatonischen, auf das >Wesen abgestellten Gattungsdenken bestimmt, das aus der Opposition von geformter (variabler) Oberfläche und formender (stabiler) Tiefe gezogen ist. Die Großprojekte der Gattungsgeschichten von Natur und Kunst stehen daher gleichzeitig und mit derselben Ratlosigkeit vor jener Frage, die u.a. die De-

${ }^{49}$ Zur Frage, inwiefern Winckelmann als `Diskursivitätsbegründer` gelten kann vgl. auch Ehrmann: Ordnen und lenken (wie Anm. 46), S. 255-258.

50 Schelling wird dies in einer seiner vielen Winckelmann-Paraphrasen mit Blick auf schwer bestimmbare kulturelle Prozesse pointieren: "Aber nicht die Kraft des Einzelnen richtet es aus; nur der Geist der sich im Ganzen verbreitet." Friedrich Wilhelm Joseph Schelling: Ueber das Verhältnis der bildenden Künste zu der Natur. Eine Rede, München 1807, S. 60 .

51 Dies in verblüffender Analogie zu Buffon: Allgemeine Historie der Natur (wie Anm. 33), bes. S. $22 f$. 
batte um Präformation und Epigenese ausgelöst hatte: ${ }^{52}$ Wie müssen die (autonom) strebenden Kräfte beschaffen sein, um aus sich gattungsbildende und zugleich evolvierende Individuen hervorbringen zu können?

Daraus wird deutlich, wie eng die poetologischen Versuche um 1800 nicht nur (wie bekannt) mit organologischen Diskursen, sondern auch mit den typologischen Modellen der sich annähernd parallel dazu institutionell konstituierenden Kunstgeschichte verbunden waren. ${ }^{53}$ Neben den alten Rangordnungen finden sich nun allerorts historisch-genealogische Stufengänge, insbesondere bei den frühen Romantikern, die emphatisch gegen die alten Hierarchien eifern. ${ }^{54}$ Dass aber gerade der Kampf gegen die "Begränzung des Individuellen« zur Formulierung überzeitlicher Urbild-Poetologien führen kann, zeigt eindrücklich das Beispiel Schellings. Für die Kunstbetrachter, die "verlangen mehr zu sehen« als das Individuum, nämlich »den lebendigen Begriff desselben", forscht der Künstler nach der »in ihm schaffenden Idea" und bildet "das Individuum zu einer Welt für sich, einer Gattung, einem ewigen Urbild $« .{ }^{55}$ Schellings Problem ist das seiner Zeit: Noch kann die äußere Gestalt nicht ohne das Vorbild einer inneren Form sedacht werden, und doch lehrt bereits der historisierende Blick, dass die individuellen Emanationen sich historisch unterscheiden, sich im Gleichschritt mit bestimmten kulturellen Bedingungen verändern und damit zugleich die folgenden Entwicklungen zu bedingen scheinen. Auch das ist eine Regel, die die Geschichte der Natur lehrt: »[E]ine Kunst, die nach allen Bestimmungen dieselbe wäre, wie die der früheren Jahrhunderte, wird nie wieder kommen; denn nie wiederholt sich die Natur. ${ }^{56}$

Dieses problematische Verhältnis von Urbild und Metamorphose lenkt den Blick unweigerlich auf Goethe, bei dem Kunst- und Naturbetrachtung noto-

52 Zur parallelen Problematik der "Ordnung des Lebendigen« und den »historischen Ordnungen der Literatur" vgl. Werner Michler: Kulturen der Gattung. Poetik im Kontext, 1750-1950, Göttingen 2015, bes. S. 74-76.

53 "Naturgeschichte und Kunstgeschichte bedingen sich in ihren Aufgaben und Funktionen gegenseitig." Reinhard Wegner: Einleitung, in: Kunst - die andere Natur, hg. von dems., Göttingen 2004, S. 7-12, hier S. 9.

$54 \mathrm{Im}$ "Versuch über den Styl in Goethes früheren und späteren Werken« aus Friedrich Schlegels Gespräch über die Poesie werden drei 'Manieren in Goethes Schaffen unterschieden, die als "Schema seines Stufenganges« bezeichnet werden. Kritische Friedrich Schlegel Ausgabe, hg. von Ernst Behler unter Mitwirkung von Jean-Jacques Anstett und Hans Eichner, I. Abt., 2. Bd., München/Paderborn/Wien 1967, S. 345.

55 Schelling: Ueber das Verhältnis der bildenden Künste zu der Natur (wie Anm. 50), S. $22 f$.

56 Ebd., S. 62. 
risch und auf produktive Weise verwoben sind. ${ }^{57}$ Nur aus dieser Konstellation gewinnt er allererst die Prämissen seiner theoretischen und praktischen Arbeit an den (künstlerischen) Gattungen. Die Vorstellung, dass jeder historischen Emanation ein bestimmter Typus zugrunde liegt, dass jedes Individuum an einer Gattung partizipiert, die es selbst zur Erscheinung bringt, leitet dabei sein Denken in beiden Bereichen. Das bekannteste Beispiel hierfür ist die in Italien entdeckte und sowohl im Versuch die Metamorphose der Pflanzen zu erklären (1790) als auch in der Elegie Die Metamorphose der Pflanzen (1798) verarbeitete `Urpflanze ${ }^{58}$ die er früh als »das wunderlichste Geschöpf von der Welt" ankündigt und die es ermöglichen soll, "noch Pflanzen ins unendliche [zu] erfinden, die konsequent seyn müßen «. ${ }^{59}$ Dabei stellt der Typus aber ein eminent unsicheres Wissen dar, der nur sgefühlt oder - und Goethe wertet diese Option zunehmend auf - induktiv aus seinen individuellen Gestaltungen erschlossen werden kann. Darum habe der Künstler kaum eine andere Wahl, als

durch Betrachtung und Übung sich auszubilden. [...] Keine Theorie giebt's, wenigstens keine allgemein verständliche, keine entschiedne Muster sind da, welche ganze Genres repräsentirten, und so muß denn jeder durch Theilnahme und Anähnlichung und viele Übung sein armes Subject ausbilden. ${ }^{60}$

Es gelte daher sowohl eine Theorie als auch einzelne Muster der Gattungen aufzustellen, ${ }^{61}$ diese damit auch voneinander zu unterscheiden und rein zu halten. ${ }^{62}$

57 Die Verbindung findet sich mit einigen Konjunkturen sein ganzes Leben lang. Die Annahme, dass sein Naturverständnis auch seine poetischen Arbeiten beeinflusst, wurde vielfach zu plausibilisieren versucht. Siehe dazu etwa Dorothea-Michaela Noé-Rumberg: Naturgesetze als Dichtungsprinzipien. Goethes verborgene Poetik im Spiegel seiner Dichtungen, Freiburg i.Br. 1993; Elisabeth von Thadden: Erzählen als Naturverhältnis - »Die Wahlverwandtschaften«. Zum Problem der Darstellbarkeit von Natur und Gesellschaft seit Goethes Plan eines »Roman über das Weltall«, München 1993; Safia Azzouni: Kunst als praktische Wissenschaft. Goethes Wilhelm Meisters Wanderjahre und die Hefte Zur Morphologie, Köln/Weimar/Wien 2005.

58 Vgl. dazu Michael Bies: Im Grunde ein Bild. Die Darstellung der Naturforschung bei Kant, Goethe und Alexander von Humboldt, Göttingen 2012, S. 162-194.

59 Goethe an Charlotte von Stein, 1.-9.7.1787, WA IV, 8, S. 232-233.

60 Goethe an Friedrich Schiller, 1.7.1797, WA IV, 12, S. 177-178.

61 Vgl. etwa Goethes Novelle, das Märchen etc. Zur Unterscheidung durch Auffinden von »Musterstücke[n] vgl. Johann Wolfgang Goethe: Naturformen der Dichtung, in: WA I, 7, S. 118-120, hier S. 119.

62 Vgl. Goethe an Friedrich Schiller, 23.12.1797, WA IV, 12, S. 382f. 
Dieses Vorhaben, das - zunehmend in Kollaborationen - die späten 1790er Jahre prägt ${ }^{63}$ wird parallel dazu von den eigenen Gattungsinnovationen (Ballade, Bildungsroman) problematisiert, ${ }^{64}$ die ein historisch-dynamisches Gattungssystem, mithin eine das organologische Denken radikalisierende Lebendigkeit implizieren. Dadurch muss jede - wenn auch mitunter selbst formulierte - Gattungshierarchie ephemer und brüchig erscheinen. ${ }^{65}$ Die 'Krise der Kunst‘ um $1800^{66}$ lässt sich daher auch als Krise der Gattungen deuten, wenn man bemerkt, dass parallel zu den Bemühungen, Kunst als einen lebendigen Organismus zu verstehen, auch ein die poetische Kraft limitierender Diskurs etabliert wurde, der die Integrität von Kunst im emphatischen Singular gewährleisten sollte. Somit ist die Frage der Gattungsreinheit zugleich eine Frage der Kunstpolitik, indem sie in das Verhältnis von Produzenten und Rezipienten, das allzu ökonomisch zu werden drohte, eingreift und die Grenze zwischen unterhaltender und ästhetischer Funktion von Kunst neu verhandelt. Die dadurch bedingte spannungsvolle, ja krisenhafte Arbeit an den Gattungen um 1800 lässt sich leicht an Goethes Propyläen veranschaulichen, die ihre eigenen gattungssystematischen Bestrebungen bereits durch die Einschaltung von Mischgattungen infrage stellen ${ }^{67}$ und sogar regelrechte Gattungshybride wie die Oper als ästhetisch besonders hochstehenden (also artifiziellen) Ausdruck `natürlicher Kunstprinzipien verhandeln. ${ }^{68}$

63 Vgl. insbesondere den 1797 nach intensiven brieflichen Diskussionen von Goethe und Schiller gemeinsam verfassten und erst 1827 in Ueber Kunst und Alterthum veröffentlichten Aufsatz Ueber epische und dramatische Dichtung.

$64 \mathrm{Zu}$ dieser Koinzidenz vgl. Michler: Kulturen der Gattung (wie Anm. 52), S. 373f.

65 Vielleicht entfaltet Goethes Text von den »Naturformen der Dichtung « auch deshalb "nicht, wie er behauptet, eine Klassifikation, sondern eine Produktionstheorie der Gattungen«. Ebd., S. 361.

66 Werner Busch: Die notwendige Arabeske. Wirklichkeitsaneignung und Stilisierung in der deutschen Kunst des 19. Jahrhunderts, Berlin 1985, S. 13f., erkennt das "Ende der Ikonographie« als Zeichen dieser Krise.

67 Siehe Johann Wolfgang Goethe: Der Sammler und die Seinigen, in: Propyläen. Eine periodische Schrift, Bd. II.2, Tübingen 1799, S. 26-122; zur Gattungsproblematik vgl. auch Norbert Christian Wolf: Vielstimmigkeit im Kontext. Goethes skleiner KunstRoman Der Sammler und die Seinigen in entstehungsgeschichtlicher und gattungstheoretischer Perspektive, in: Klassizismus in Aktion (wie Anm. 22), S. 239-276.

68 Siehe Goethe: Ueber Wahrheit und Wahrscheinlichkeit der Kunstwerke (wie Anm. 25). 
IV.

Wenig spricht nunmehr dagegen, dass unterschiedliche literarische Gattungen ebenso aus untergegangenen Vorgängern hervorgehen können ${ }^{69}$ wie »der gemeine und indische Stier" aus jener "weit verbreitete[n] untergegangene[n] Stamm-Race«, als die Goethe einen fossilen Stier anerkennt. ${ }^{70}$ In analoger Weise gehen daher die drei in Goethes Noten und Abhandlungen zum Diwan (1819) eingeführten Redegattungen der Poesie (Epos, Lyrik und Drama) aus einer - wenn auch namenlosen - >Stamm-Race hervor; sie bilden zunächst einen Kollektivkörper »und erst in einer gewissen Zeitfolge sondern sie sich. ${ }^{71}$ Die Dreiteilung ist zwar unabweislich eine transhistorisch gattungssystematische, jedoch soll sie - dem vorangehenden, "Dichtarten« betitelten Abschnitt der Noten zufolge - auch »historischen Zwecken « dienen können. ${ }^{72}$ In Anerkennung der potenziellen phylogenetischen Transformationen der Dichtungsarten muss, wer von `Naturformen` spricht, auch mit dem Abnormen kalkulieren. Diese jede Taxonomie gefährdenden Anomalien sind indes "nicht gleich als krank, oder pathologisch zu betrachten ${ }^{73}$ und als solche außerhalb der Ordnung zu platzieren, sie weisen vielmehr auf das "unsichere Vor- und Rückschreiten der Natur« hin. ${ }^{74}$ Es sind beide, "sowohl das Geregelte als Regellose, von einem Geiste belebt«, und es überschreitet die Natur im Abnormen zwar »die Gränze, die sie sich selbst gesetzt hat, aber sie erreicht dadurch eine andere Vollkommenheit«. ${ }^{75}$ Naturformen bringen daher auch im Bereich der Dichtung Ränder mit sich, an denen Deviantes, nicht aber Pathologisches erscheint. Die behauptete Reinheit der Gattungen ist eine hypothetische, die durch Musterwerke zwar insinuiert, historisch aber kaum gegenüber kontaminierenden Textpraktiken aufrechterhalten werden kann.

69 Charles Darwin wird später das Aussterben als notwendige Voraussetzung von Gattungsdifferenzierung betrachten; vgl. Philipp Sarasin: Darwin und Foucault. Genealogie und Geschichte im Zeitalter der Biologie, Frankfurt a.M. 2009, S. 135.

70 Johann Wolfgang Goethe: Fossiler Stier, in: WA II, 8, S. 234; vgl. dazu auch Margrit Wyder: Goethes Naturmodell. Die Scala Naturae und ihre Transformationen, Köln/Weimar/Wien 1998, S. $276 f$.

71 Goethe: Naturformen der Dichtung (wie Anm. 61), S. 118.

72 Johann Wolfgang Goethe: Dichtarten, in: WA I, 7, S. 117.

73 Johann Wolfgang Goethe: Zur Morphologie (Nacharbeiten und Sammlungen), in: WA II, 6, S. 169-186, hier S. 173.

74 Ebd., S. 172.

75 Ebd., S. 174. 
Gerade um solche wilden, dem emphatischen Kunstdiskurs des frühen 19. Jahrhunderts so zuwiderlaufenden und ihn dennoch so nachhaltig faszinierenden Praktiken scheint es sich bei den zeitgleich auftauchenden Urformen zu handeln, die sich nicht zuletzt aus dem Bereich der vernakulären >Volks-SDichtung speisen. Einst populäre, hochgradig unsichere und bisweilen unreine Textpraktiken werden in teils aufwendigen Ursprungserzählungen an den Anfang der Gattungsgeschichte gesetzt. Für den Zusammenhang von Lebens- und Gattungswissen besonders aufschlussreich ist Goethes bekannter Text über die Ballade, der wohl nicht als die programmatische Äußerung konzipiert war, als die er wahrgenommen wurde. Zumal der kurze Text - seltsam anachronistisch - erst 1821 im ersten Heft des dritten Bandes von Goethes Zeitschrift Ueber Kunst und Alterthum erschienen ist - und damit Jahrzehnte nach der 'Erfindung der Ballade als Kunstform. Jedoch übernimmt die relativ junge Gattung der Ballade um 1820 offenbar eine geänderte Funktion; sie dient nicht mehr dazu, die poetogene Kraft des Volkes zu beschwören und für die Kunst fruchtbar zu machen, sondern ist zum programmatischen Ort der Gattungsreflexion geworden. Daher figuriert sie bereits in den Naturformen der Dichtung als »das herrlichste Gebild«, das durch die "Vereinigung « der drei `Naturformen »im engsten Raume « entsteht. ${ }^{76}$ Wenngleich der Balladen-Text dieses Verschmelzungsmotiv aufgreift und im Bild vom »lebendige[n] Ur-Ey « pointiert, ${ }^{77}$ scheint er die Programmatik des Naturformen-Aufsatzes sukzessive zurückzunehmen. Das zeigt sich nicht zuletzt an der Druckgeschichte: Denn er wurde zunächst als erstes Stück der Nachträge zu den vorigen Heften und sonstige Einzelnheiten unter dem Titel Ballade. Betrachtung und Auslegung veröffentlicht, erschien im (postumen) 45. Band der Ausgabe letzter Hand indes als Ueber die Ballade vom vertriebenen und zurückkehrenden Grafen. ${ }^{78}$ Die Veränderung ist bedeutender, als es auf den ersten Blick scheinen mag. Immerhin stellt der Text einen Kommentar zu der im vorangegangenen Heft von Ueber Kunst und Alterthum erschienenen Ballade Ballade dar, der von einigen allgemeinen Bemerkungen eingeleitet wird. Der eben nicht unbetitelte, sondern in der Titelwahl eine exemplarische Gattungsnorm verheißende Bezugstext wird in der Ausgabe letzter

76 Goethe: Naturformen der Dichtung (wie Anm. 61), S. 118.

77 Johann Wolfgang Goethe: Ballade. Betrachtung und Auslegung, in: Ueber Kunst und Alterthum, Bd. III.1, Stuttgard [!] 1821, S. 49-55, hier S. 50.

78 Johann Wolfgang Goethe: Ueber die Ballade vom vertriebenen und zurückkehrenden Grafen, in: Goethe's Werke. Ausgabe letzter Hand, Bd. 45, Stuttgart/Tübingen 1833, S. 332-336. 
Hand durch den inhaltsbeschreibenden Zusatz individualisiert. Aus dem Gattungsmodell wird ein Gattungsexemplar.

Diese Tendenz lässt sich auch im Text selbst feststellen, der die Emphase der Naturanalogie im Naturformen-Aufsatz insgesamt abzumildern scheint. Der Zusammenfall der Dichtarten ist nicht mehr mit der Primordialität der Ballade verknüpft, ${ }^{79}$ sondern den Zufälligkeiten der dichterischen (oder vernakulär-poetischen?) Praxis geschuldet. ${ }^{80}$ Die Ballade wird nämlich performiert von einem Sänger, der »nicht weiß wie er ihn [seinen prägnanten Gegenstand] ans Tageslicht fordern will. ${ }^{81}$ Keine naturgleiche Konsequenz regelt die Vermischung der 'Elementes, sondern die Medialität der Aufführung; die Ballade ist daher weniger Natur- als - "nach Belieben die Formen wechselnd $\aleph^{82}-$ Sozialform.

Da der Balladen-Text - insbesondere im Erstdruck - dezidiert auf die programmatisch betitelte und die Gattungsmischung tatsächlich durchexerzierende Ballade Bezug nimmt, ${ }^{83}$ muss sie notwendig in eine Analyse des darin verhandelten Lebenswissens miteinbezogen werden. ${ }^{84}$ Die Ballade beginnt mit einer vom späteren Balladen-Text betonten Reflexion der Redesituation, indem zunächst die Kinder jenen Alten hereinbitten, der ab der zweiten Strophe fast ausschließlich das Wort haben und der "Sänger" des folgenden Textes sein wird. ${ }^{85}$ Ab der sechsten Strophe wird das Verhältnis des Sängers zu seinem Text wiederum prekär, da er die ('episch`) berichtende Haltung aufgibt, um selbst als (lyrisches`) 'Ich zu sprechen, während in der Folge seine Handlungen wiederum aus Berichtsperspektive geschildert werden. ${ }^{86}$ Dabei ist es ihm nach dem Bruch der sechsten Strophe nicht mehr möglich,

79 Aufgabe der Ballade scheint es also durchaus zu sein, das Programm des berühmten 116. Athenäums-Fragments umzusetzen und »alle getrennte Gattungen der Poesie wieder zu vereinigen«. Vgl. Kritische Friedrich Schlegel Ausgabe (wie Anm. 54), S. 182.

80 Das ist gemäß der mit Schiller geführten gattungspoetologischen Debatten ein gewichtiges Problem für die Produktivität einer Gattung: »Eine reine Form hilft und trägt, da eine unreine überall hindert und zerrt." Goethe an F. Schiller, 30.10.1797, WA IV, 12, S. 352.

81 Goethe: Ballade. Betrachtung und Auslegung (wie Anm. 77), S. 49.

82 Ebd., S. 50.

83 Vgl. ebd., S. 51.

84 Es ist sehr befremdlich, dass Ballade. Betrachtung und Auslegung in der Forschung fast durchwegs als eigenständig behandelt und von ihrem Bezugstext getrennt wird. Die Ballade wurde schon zeitgenössisch wenig geschätzt. Wie aus Eckermanns Gesprächen mit Goethe, 16.12.1828, MA 19, S. 274, hervorgeht, hat »das deutsche Publikum bis jetzt nicht viel daraus [...] machen können".

85 Vgl. Johann Wolfgang Goethe: Ballade, in: Ueber Kunst und Alterthum, Bd. II.3, Stuttgard [!] 1820, S. 7-12, hier S. 7.

86 Vgl. ebd., S. 10; vgl. auch Goethe: Ballade. Betrachtung und Auslegung (wie Anm. 77), S. 52 . 
unmarkiert die Position des Berichterstatters eines nunmehr gegenwärtigen Geschehens einzunehmen. Dieses Problem liegt nun freilich weniger im Zusammenstoßen heterogener 'Naturformen komplexen Verhältnis von Textualität und Medialität.

Dabei verhandelt die Ballade eine im neuen Lebenswissen durchaus virulente Thematik: Denn nachdem sich der bettelnde Sänger als der Graf seiner eigenen Erzählung entpuppt hat, setzt eine eigenartige (’dramatische) Szene ein, die spannungsreich die Frage der Vererbung thematisiert. Der heimkehrende Vater möchte den vermeintlichen Bettler ins Verließ werfen lassen. Als seine Frau - die Tochter des nunmehr gräflichen Sängers - für diesen bittet, beginnt er sie und seine Kinder im Bewusstsein seiner "alten ritterlichen Herkunft ${ }^{87} \mathrm{zu}$ beschimpfen: "Du niedrige Brut! du vom Bettlergeschlecht! / Verfinsterung fürstlicher Sterne! / Ihr bringt mir Verderben! Geschieht mir doch Recht $-\ll{ }^{88}$ Die topische Ständehierarchie wird hier auch als biologische aufgerufen. Die Reihe 'Brut $<$ - 'Geschlecht $<$ - >Verderben ruft Degenerationsphantasien auf, die indes weniger adeligen Angstträumen als vielmehr den kunsttheoretischen Debatten der Jahrhundertwende entstammten. Bereits Goethes Einleitung in die Propyläen führt als "[e]ines der vorzüglichsten Kennzeichen des Verfalles der Kunst [...] die Vermischung der verschiedenen Arten derselben « an. ${ }^{89}$ Der Ritter geht aber noch weiter: "Schon lange verflucht' ich mein ehliches Glück, / Das sind nun die Früchte der Blüthen! / Man leugnete stets, und man leugnet mit Recht, / Daß je sich der Adel erlerne / Die Bettlerinn zeugte mir Bettlergeschlecht $-\ll{ }^{90}$ Die Vermischung zeigt keinen Niedergang an, sondern löscht - vermeintlich! - den in emphatischer Reinheit begriffenen Adel bereits in der folgenden Generation aus. Der Wert der Ahnentafel wird damit sowohl von außen durch die seit dem Ende des Alten Reichs deutlich vermehrten Nobilitierungen als auch von innen durch Mesalliancen gefährdet. Diese Kalamität des Adels zu Beginn des 19. Jahrhunderts führt zu der erwartbaren Krise seiner Begründung in einfachen Vorstellungen von Gottesgnadentum und Präformation, aber auch zur Integration epigenetischer Denkmodelle in das eigene Legitimationssystem. ${ }^{91}$ Wenn daher die Verbindung von Adel und

87 Ebd., S. 53.

88 Goethe: Ballade (wie Anm. 85), S. 11.

89 Johann Wolfgang Goethe: Einleitung, in: Propyläen. Eine periodische Schrift, Bd. I.1, Tübingen 1798, S. III-XXXVIII, hier S. XXIV.

90 Goethe: Ballade (wie Anm. 85), S. 11.

91 Es soll hier aber keine gewissermaßen natürliche Nähe von Adel und Präformation bzw. umgekehrt von Bürgertum und Epigenesis insinuiert werden. Es geht nicht um 'moderner 
Bettler nicht wiederum Adel hervorbringen kann, dann handelt es sich nach der Methodik der Artunterscheidung des (Grafen) Buffon, den Goethe in seinen letzten Lebensjahren auch aus publikationsstrategischem Interesse gerne "nach Tische ${ }^{92}$ las - um verschiedene Arten. ${ }^{93}$ Die Vermischung der Stände muss daher am Ende zur ,Verfinsterung fürstlicher Sterne‘, zur Vermehrung des 'Bettlergeschlechts` führen; das ist die Kehrseite des ständischen Reinheitsgebots, das auch von der Schlusswendung nicht aufgehoben, sondern nur ins Positive gezogen wird. Der alte Graf, dessen adelige ’Form und Physiognomie offenbar auch von Standesgenossen nicht erkannt werden konnte, löst die Verwechslung auf und beruhigt seinen (ihm nunmehr in der Adelshierarchie unterlegenen) Schwiegersohn: »Erhole dich, Sohn! Es entwickelt sich gut, / Heut einen sich selige Sterne, / Die Fürstin sie zeugte dir fürstliches Blut $-\ll{ }^{94}$ Innerhalb weniger Verse werden die Kinder von Bettlern zu Fürsten und geraten damit selbst zum Verweis auf das Fehlen einer gesicherten Wahrnehmbarkeit von Nobilität. Durch die heikle Verschiebung der `Mischerzeugnisse ‘ auf der Ständeleiter wird die Biologie der Fortzeugung des Adels in den Bereich des Nichtwissens versetzt - und es ist freilich gerade dies der Ort, an dem die biologische Begründung überhaupt erfolgen kann. Der Graf, der nicht nur die Enkel ins Recht setzt, sondern unter der Hand zugleich den Adel vom Erbstand zum Sprechakt macht, schließt mit dem letzten Refrain: "Die Kinder sie hören es gerne. ${ }^{95}$ Wer sonst?

Blut anstatt Brut ist das Ergebnis der stellaren Vereinigung. Diese biologische Verfestigung des ständischen Systems in einer selbst Formen des Natürlichen verhandelnden Dichtung sollte nicht kurzschlüssig auf das

Herausforderungen rückständiger Systeme und das Aufbieten anschaulicher Überwindungsszenarien; vielmehr verweist diese Krise auch auf die unübersichtliche Gemengelage von Zeugungs- und Entwicklungstheorien im 18. und frühen 19. Jahrhundert. Vgl. dazu Stefan Borchers: Die Erzeugung des "ganzen Menschen«. Zur Entstehung von Anthropologie und Ästhetik an der Universität Halle im 18. Jahrhundert, Berlin/New York 2011, S. 1-17.

92 Goethe Tagebuch, 8. und 9.8.1830, WA III, 12, S. $285 f$.

93 "[Z]u einer Art seyen nur die Thiere zu rechnen, die sich beständig, unabläßig, unveränderlich, kurz, auf die Art, wie andere Thiere fortpflanzen." Buffon: Allgemeine Historie der Natur (wie Anm. 33), S. 9.

94 Goethe: Ballade (wie Anm. 85), S. 12. Es handelt sich dabei also gerade nicht um den Ausdruck eines »bürgerliche[n] Prinzip[s] von Liebe und Familie, das nicht nach Herkunft fragt«, wie Matthias Mayer: "Die Kinder sie hören es gerne." Politik und Poetik in Goethes »Ballade«, in: Jahrbuch des Freien Deutschen Hochstifts 1987, S. 60-94, hier S. 74, meint.

95 Goethe: Ballade (wie Anm. 85), S. 12. 
kompensatorische Verhalten eines (nur) nobilitierten Dichters zurückgeführt werden. Denn die epigenetischen Entwicklungsszenarien bedrohten die Kunst im emphatischen Singular ebenso sehr wie den Adel. Wie dieser vom Bettlergeschlecht wird die Kunst - wie man jetzt sah - seit der Antike vom Handwerk bedroht. ${ }^{96}$ Der Gegensatz von Fertigen und Schaffen gewinnt durch die schrittweise Etablierung der industriellen Produktion eine Aktualität, deren Brisanz im Laufe des 19. Jahrhunderts noch zunimmt. ${ }^{97}$ Immer weiter entfernt sich so das mühsam geschaffene Kunstwerk vom Handwerk, das sich zu einem nach Matrizen gefertigten Konsumgut steigern lässt. ${ }^{98}$ In der Abwertung der stoten< Handwerksgüter zeigt sich erneut und nachdrücklich die Macht des biologischen Interdiskurses. Die über biologisches Wissen gespielte Konzeption reiner Gattungen soll bei Goethe die Kunst vor degenerativer Vermischung und dem Abstieg zu Unterhaltung oder Handwerk bewahren. ${ }^{99}$ Am Ende wird Lebenswissen bei Goethe zu Kunstpolitik: Bald schon arbeitete er vormittags an seiner Ausgabe letzter Hand, die aus dem programmatischen Ort poetischer Gattungsreflexion die Geschichte »vom vertriebenen und zurückkehrenden Grafen« machen wird; nach Tische las er »in Buffon weiter ${ }^{100}$

96 Vgl. GKA, 235f.: »Die Nachahmung beförderte den Mangel eigener Wissenschaft, wodurch die Zeichnung furchtsam wurde, und was der Wissenschaft abgieng, suchte man durch Fleiß zu ersetzen, welcher sich nach und nach in Kleinigkeiten zeigete, die in den blühenden Zeiten der Kunst übergangen, und dem großen Stile nachtheilig geachtet worden sind."

97 Hartmut Winkler: Technische Reproduktion und Serialität, in: Endlose Geschichten. Serialität in den Medien, hg. von Günter Giesenfeld, Hildesheim 1994, S. 38-45, hier S. 38, hat bemerkt, dass »die industrielle Massenherstellung von Konsumgütern, [...] nur auf der Basis des seriellen Prinzips überhaupt denkbar [ist]《.

98 In der kleinstädtischen Enge Weimars kommen beide Extreme nebeneinander zu liegen: Eine bis zur Immobilität gesteigerte ästhetische Autonomietheorie trifft dort auf Friedrich Justin Bertuchs sehr volatil an Opportunem orientiertes fournal des Luxus und der Moden und dessen praktische Verlängerung, das ebenfalls von dem geschäftstüchtigen Legationsrat betriebene Landes-Industrie-Comptoir. Vgl. Friedrich Justin Bertuch (1747-1822): Verleger, Schriftsteller und Unternehmer im klassischen Weimar, hg. von Gerhard R. Kaiser und Siegfried Seifert, Tübingen 2000; Walter Steiner/Uta Kühn-Stillmark: Friedrich Justin Bertuch. Ein Leben im klassischen Weimar zwischen Kultur und Kommerz, Köln/Weimar/Wien 2001.

99 In einem Paralipomenon zum Beitrag über Vorbilder für Fabricanten und Handwerker für den dritten Band von Ueber Kunst und Alterthum weist Goethe auf die gefährliche Nähe vom "untergeordneten Künstler« zum »kunstreichen Handwerker« hin (WA I, 49.2, S. 271).

${ }^{100}$ Goethe Tagebuch, 9.8.1830, WA III, 12, S. 286. 
